\title{
Critical behavior for the dilaton black holes
}

\author{
Rong-Gen Cai ${ }^{a, b, *}$ and Y. S. Myung ${ }^{c}$ \\ a CCAST (World Laboratory), P.O. Box 8730, Beijing 100080, China \\ ${ }^{b}$ Institute of Theoretical Physics, Academia Sinica, P.O. Box 2735, Beijing 100080, China \\ ${ }^{c}$ Department of Physics, Inje University, Kimhae 621-749, Korea
}

\begin{abstract}
We study the critical behavior in the black $p$-branes and four dimensional charged dilaton black holes. We calculate the thermodynamic fluctuations in the various (microcanonical, canonical, and grandcanonical) ensembles. It is found that the extremal limit of some black configurations has a critical point and a phase transition takes place from the extremal to nonextremal black configurations. Some critical exponents are obtained, which satisfy the scaling laws. This is related to the fact that the entropy of these black configurations is a homogeneous function.
\end{abstract}

PACS numbers: 04.70.Dy, 05.40.+j, 05.70.Fh, 64.60.Fr, 04.50.+h

Keywords: Phase transition, Critical exponents, Black $p$-branes, Dilaton black holes

Typeset using REVTEX

*E-mail: cairg@itp.ac.cn 


\section{INTRODUCTION}

The Hawking radiation for a black hole is one of the most important achievements of quantum field theory in curved spacetime [1]. It resolved the puzzle of the mathematical analogy between the black hole mechanics and thermodynamics [2]. Further it offered the basic concept of black hole entropy suggested by Bekenstein [3]. The establishment of temperature and entropy for a black hole implies that the laws of thermodynamics can be applied to understand a black hole [4]. However there are some important differences between black holes and ordinary thermodynamic systems. For examples, the Hawking-Bekenstein area formula for the black hole entropy is not an extensive quantity; some black holes have negative heat capacities; the system containing a black hole has only a local equilibrium temperature, which diverges at the black hole horizon. Unlike the ordinary thermodynamic system, black hole is not a uniform system. This means that a black hole cannot be divided into some subsystems. These differences give rise to some difficulties in studying the black hole thermodynamics. Hence it is of interest to investigate the phase transition in black hole thermodynamics.

Nowadays there exist two approaches to the phase transition of black holes. One comes form the work of Davies [5]. He argued that a second-order phase transition can take place at the singular points of heat capacity of black holes. Many authors have discussed the singular points for various black holes $[6,7,8,9,10]$. Lousto [9] claimed that the singular points satisfy the scaling laws of critical points. In particular, an effective spatial dimension of black holes is obtained as two. Further he insisted that his result agrees with the membrane picture

of black holes [11]. Later on, it turns out that his calculation is wrong and the effective spatial dimension of black holes is not two. It seems that all discussions on his effective two-dimensional quantum description of black holes become invalid.

On the other hand, the other group considered that a phase transition of black holes occurs when a nonextremal black hole approaches its extremal counterpart. This was first reported by Curir [12]. Pavón and Rubí [13] found that all second moments of nonequilibrium 
fluctuations are finite for the Schwarzschild black hole with single horizon, but some second moments diverge for the extremal Kerr black hole and extremal Reissner-Nordström black hole. The divergence of second moments means that a second-order phase transition takes place when the nonextremal black hole is turned into the extremal one. An important point to remark is that all second moments have no any special behavior and are finite at the points of Davies. Cai, Su, and Yu [14] obtained the same result in the charged dilaton black holes and Kerr-Newman black holes. However, all second moments are finite for the $a=1$ charged dilaton black holes. So the difference of outer horizon and inner horizon plays an important role in the divergence of second moments, because the $a=1$ charged dilaton black hole, as the Schwarzschild black hole, has a single horizon.

Davies' phase transition (that is, due to the discontinuity of heat capacity) is not so attractive. This is because the event horizon does not lose its regularity and the internal states of black holes are not changed significantly through the Davies' points [7]. On the other hand, it is more interesting to study the phase transition between the extremal and nonextremal black holes. As is well known, the extremal black holes have vanishing Hawking temperature. Therefore, the Hawking evaporation is terminated and only the superradiation is left for an extremal black hole. The geometric structure of extremal black holes is also different with nonextremal black holes, since the singularity will be naked beyond the extremal limit. Wilczek and his collaborators [15] argued that the thermodynamic description is inadequate for some extremal black holes and their behavior resembles the normal elementary particles, strings, or extended objects. In addition, Kaburaki and his collaborators [16] found that the Davies' points in fact are turning points, which are related to the changes of stability. Also they claimed that the divergence of heat capacity at those points does not mean the occurrance of phase transition. More recently, Kaburaki [17] discussed the equilibrium thermodynamic fluctuations of Kerr-Newman black hole in various ensembles. He found that the extremal Kerr-Newman black hole has a critical point and some critical exponents also obey the scaling laws [18].

In the previous work [19], by investigating the critical behavior and phase transition 
in the $2+1$ dimensional black holes recently found by Bañados, Teitelboim, and Zanelli (BTZ) [20], we obtained some interesting results: (1) The extremal spinning BTZ black hole has a critical point and a second-order phrase transition takes place from extremal to nonextremal one. (2) The massless BTZ black hole has also a critical point and a secondorder phase transition occurs from a massless to massive one. (3) Some critical exponents remain unchanged (as those of Kerr-Newman black hole) and satisfy the scaling laws. (4) In particular, the effective spatial dimension of BTZ black hole is found to be one. This supports that extremal black holes are the Bogomol'nyi saturated string states.

On the basis of above results, some questions arise as follows. Are these critical exponents of the extremal black holes universal? Is the effective spatial dimension of other black holes also one? Could we obtain a reduced quantum model of black holes? In the present paper, we try to reply in part to the first two questions by extending our discussion to the black

p-branes and four-dimensional dilaton black holes with (and without) moduli field. We note that the thermodynamic properties of these black configurations are significantly affected by the dilaton and moduli fields.

The organization of this paper is as follows. We first introduce the black $p$-branes, and then discuss their thermodynamic properties in Sec. II. In Sec. III we investigate the equilibrium fluctuations of black p-branes in the microcanonical ensemble, canonical ensemble, and grand canonical ensemble. Further we calculate some relevant critical exponents. We discuss the case of the four-dimensional charged dilaton black holes in Sec. IV. In Sec. V we study the effects of a moduli field on the critical behavior of charged dilaton black holes. Finally we conclude in Sec. VI with a brief discussion.

\section{BLACK STRINGS AND P-BRANES AND THEIR THERMODYNAMIC PROPERTIES}




\section{A. Black strings and Black $p$-branes}

The $p=1$ black string and $p$-brane solutions are relevant to black hole physics [21]. These are some extended objects surrounded by event horizons. In this paper, we have $p=\tilde{d}-1$ for a "magnetically" charged object and $p=d-1$ for a "electrically" charged one. As black holes in general relativity, these extended objects in the string theories have thermodynamic properties, such as the entropy and Hawking temperature, etc. It is expected that these extended objects might play the important roles in understanding the fundamental nature of sting theory and quantum gravity. It was shown that they are solitonic solutions [22], and are closely related to fundamental strings and membranes themselves [21]. More recently, it appears that the Bekenstein-Hawking entropy of extremal, near-extremal black holes, and black $p$-branes agrees with the string entropy derived from the bound states of Dirichlet branes [23]. Therefore, it is important to discuss the critical behavior for these extremal black extended objects.

We start with the R-R sector of supergravity action $[21,22,24]$

$$
I_{\mathrm{D}}=\frac{1}{16 \pi} \int d^{D} x \sqrt{-g}\left(R-\frac{1}{2}(\nabla \phi)^{2}-\frac{e^{-\alpha(d) \phi}}{2(d+1) !} F_{d+1}^{2}\right)-\frac{1}{8 \pi} \int d^{D-1} x \sqrt{-h} K,
$$

where

$$
\alpha^{2}(d)=4-\frac{2 d \tilde{d}}{d+\tilde{d}}, \quad \tilde{d}=D-d-2
$$

Here $R$ is the scalar curvature, $\phi$ is the dilaton field, $F_{d+1}$ denotes the $(d+1)$-form tensor field and $K$ the extrinsic curvature on the induced metric $h$. Varying the action (1), one finds the equations of motion

$$
\begin{aligned}
& R_{\mu \nu}-\frac{1}{2} g_{\mu \nu} R=\frac{1}{2} \nabla_{\mu} \phi \nabla_{\nu} \phi+\frac{1}{2 d !} e^{-\alpha \phi} F_{\mu \ldots} F_{\nu} \ldots \\
& -\frac{1}{4} g_{\mu \nu}\left((\nabla \phi)^{2}+\frac{1}{(d+1) !} e^{-\alpha \phi} F_{d+1}^{2}\right) \\
& \nabla^{2} \phi=\frac{-\alpha}{2(d+1) !} e^{-\alpha \phi} F_{d+1}^{2} \\
& 0=\nabla^{\mu}\left(e^{-\alpha \phi} F_{\mu \ldots \nu}\right) .
\end{aligned}
$$


We wish to obtain the general $(\tilde{d}-1)$-brane solution for the action (1). First we require that the solution is invariant under the symmetry group $R \times S O(d+2) \times E(\tilde{d}-1)$, where $E(n)$ denotes the $n$-dimensional Euclidean group. This is realized as

$$
d s^{2}=e^{2 X} d \hat{s}^{2}+e^{2 Y} d x^{i} d x^{i},
$$

where $d \hat{s}^{2}$ is a $(d+3)$-dimensional Lorentzian line element, $x^{i}$ are external coordinates for $(\tilde{d}-1)$-dimensions. All fields and functions $X$ and $Y$ are independent of $x^{i}$ and therefore the solution is invariant under the translational and rotational transformations in $(\tilde{d}-1)$ dimensions. Substituting (5) into (1), we obtain the action per unit volume for the $(\tilde{d}-1)$ brane

$$
I_{d+3}=\frac{1}{16 \pi} \int d^{d+3} x \sqrt{-g}\left(R-\frac{1}{2}(\nabla \rho)^{2}-\frac{1}{2}(\nabla \sigma)^{2}-\frac{1}{2(d+1) !} e^{\beta \rho} F_{d+1}^{2}\right),
$$

where the $\rho$ and $\sigma$ are defined by the relation

$$
\begin{aligned}
& \beta X=-\frac{d(\tilde{d}-1)}{(d+\tilde{d})(d+1)} \rho+\sqrt{\frac{\tilde{d}-1}{2(d+\tilde{d})(d+1)}} \alpha(d) \sigma, \\
& \beta Y=\frac{d}{d+\tilde{d}} \rho-\sqrt{\frac{d+1}{2(d+\tilde{d})(\tilde{d}+1)}} \alpha(d) \sigma, \\
& \beta \phi=-\alpha(d) \rho-\frac{2 d}{\sqrt{2(d+\tilde{d})}} \frac{\tilde{d}-1}{d+1} \sigma,
\end{aligned}
$$

and the constant $\beta$ is given by

$$
\beta=-\sqrt{\frac{2(d+2)}{d+1}} .
$$

The problem of finding black ( $\tilde{d}-1)$-brane solution of (1) is thereby reduced to the problem of finding black hole solution of (6). The asymptotically flat and spherically symmetric black hole solutions for the action (6) were found in [25] as

$$
\begin{aligned}
F & =Q_{m} \epsilon_{d+1}, \\
d \hat{s}^{2} & =-\left[1-\left(\frac{r_{+}}{r}\right)^{d}\right]\left[1-\left(\frac{r_{-}}{r}\right)^{d}\right]^{1-\gamma d} d t^{2}+\left[1-\left(\frac{r_{+}}{r}\right)^{d}\right]^{-1}\left[1-\left(\frac{r_{-}}{r}\right)^{d}\right]^{\gamma-1} d r^{2} \\
& +r^{2}\left[1-\left(\frac{r_{-}}{r}\right)^{d}\right]^{\gamma} d \Omega_{d+1}^{2},
\end{aligned}
$$




$$
\begin{aligned}
e^{\beta \rho} & =\left[1-\left(\frac{r_{-}}{r}\right)^{d}\right]^{\gamma d}, \\
\sigma & =0
\end{aligned}
$$

where $\epsilon_{d+1}$ is the volume element of the unit $(d+1)$-sphere and the constant $\gamma$ is

$$
\gamma=\frac{d+2}{d(d+1)}
$$

Here the constant $Q_{m}$ is the magnetic charge of the black hole defined by two parameters $r_{-}$and $r_{+}\left(\geq r_{-}\right)$,

$$
Q_{m}=d\left(r_{-} r_{+}\right)^{d / 2}
$$

The structure of solutions (7)-(10) is clear. The parameter $r_{+}$corresponds to the event horizon of the black hole, while $r_{-}$is a scalar curvature singularity. It is straightfoward to show that the curvature $R$ is finite at $r=r_{+}$. From the last term in (8) with $\gamma>0$, at $r=r_{-}$the area of the $(d+1)$-sphere goes to zero and thus $R$ is singular. In fact $r=r_{-}$is a spacelike surface of singularity, very similar to the singularity in the Schwarzschild metric. Using (5)-(10), we obtain the black $(\tilde{d}-1)$-brane solutions for the action (1)

$$
\begin{aligned}
& F=Q_{m} \epsilon_{d+1}, \\
& e^{-2 \phi}=\left[1-\left(\frac{r_{-}}{r}\right)^{d}\right]^{\gamma_{\phi}}, \\
& d s^{2}=-A(r) d t^{2}+B(r) d r^{2}+r^{2} C(r) d \Omega_{d+1}^{2}+D(r) d x^{i} d x^{i},
\end{aligned}
$$

where

$$
\begin{aligned}
& A(r)=\left[1-\left(\frac{r_{+}}{r}\right)^{d}\right]\left[1-\left(\frac{r_{-}}{r}\right)^{d}\right]^{\gamma_{x}-1}, \\
& B(r)=\left[1-\left(\frac{r_{+}}{r}\right)^{d}\right]^{-1}\left[1-\left(\frac{r_{-}}{r}\right)^{d}\right]^{\gamma_{\Omega}-1}, \\
& C(r)=\left[1-\left(\frac{r_{-}}{r}\right)^{d}\right]^{\gamma_{\Omega}}, \\
& D(r)=\left[1-\left(\frac{r_{-}}{r}\right)^{d}\right]^{\gamma_{x}},
\end{aligned}
$$

and

$$
\gamma_{x}=\frac{d}{d+\tilde{d}}, \quad \gamma_{\Omega}=\frac{\alpha^{2}(d)}{2 d}, \quad \gamma_{\phi}=\alpha(d)
$$


This is a "magnetically" charged black $(\tilde{d}-1)$-brane solution with two parameters $r_{-}$and $r_{+}$. To obtain the black $p$-brane solutions with "electric" charge, we dualize these forms as in [21]. The equation of motion of $F_{d+1}$ in (4) implies that the $(\tilde{d}+1)$-form $\tilde{F}_{\tilde{d}+1}=e^{-\alpha \phi} * F_{d+1}$

is closed, where $*$ denotes the Hodge dual with $(*)^{2}=1$. If one replaces $F_{d+1}^{2}$ by $\tilde{F}_{\tilde{d}+1}^{2},-\alpha$ by $\alpha$, and $(d+1)$ by $(\tilde{d}+1)$, the action (1) then becomes

$$
\tilde{I}_{\mathrm{D}}=\frac{1}{16 \pi} \int d^{D} x \sqrt{-g}\left(R-\frac{1}{2}(\nabla \phi)^{2}-\frac{e^{\alpha \phi}}{2(\tilde{d}+1) !} \tilde{F}_{\tilde{d}+1}^{2}\right)-\frac{1}{8 \pi} \int d^{D-1} x \sqrt{-h} K .
$$

Considering the action (14), we obtain the equations of motion

$$
\begin{aligned}
R_{\mu \nu} & -\frac{1}{2} g_{\mu \nu} R=\frac{1}{2} \nabla_{\mu} \phi \nabla_{\nu} \phi+\frac{1}{2 \tilde{d} !} e^{\alpha \phi} \tilde{F}_{\mu \ldots} \tilde{F}_{\nu} \ldots \\
& -\frac{1}{4} g_{\mu \nu}\left((\nabla \phi)^{2}+\frac{1}{(\tilde{d}+1) !} e^{\alpha \phi} \tilde{F}^{2}\right), \\
\nabla^{2} \phi & =\frac{\alpha}{2(\tilde{d}+1) !} e^{\alpha \phi} \tilde{F}^{2}, \\
0 & =\nabla^{\mu}\left(e^{\alpha \phi} \tilde{F}_{\mu \ldots \nu}\right) .
\end{aligned}
$$

Comparing Eqs.(15)-(17) with Eqs. (2)-(4), it is easy to find that Eqs. (15)-(17) are exactly identical with Eqs. (2)-(4), after replacing $\tilde{F}_{\tilde{d}+1}^{2}$ by $F_{d+1}^{2}, \alpha$ by $-\alpha$, and $\tilde{d}$ by $d$. Thus one can obtain the "electrically" charged black $(d-1)$-brane solutions for the action (1),

$$
\begin{aligned}
& \tilde{F}_{\tilde{d}+1}=\tilde{d}\left(r_{+} r_{-}\right)^{\tilde{d} / 2} \epsilon_{\tilde{d}+1} \\
& e^{-2 \phi}=\left[1-\left(\frac{r_{-}}{r}\right)^{\tilde{d}}\right]^{\tilde{\gamma}_{\phi}}, \\
& d s^{2}=-\tilde{A}(r) d t^{2}+\tilde{B}(r) d r^{2}+r^{2} \tilde{C}(r) d \Omega_{\tilde{d}+1}^{2}+\tilde{D}(r) d x^{j} d x^{j},
\end{aligned}
$$

where

$$
\begin{aligned}
& \tilde{A}(r)=\left[1-\left(\frac{r_{+}}{r}\right)^{\tilde{d}}\right]\left[1-\left(\frac{r_{-}}{r}\right)^{\tilde{d}}\right]^{\tilde{\gamma}_{x}-1}, \\
& \tilde{B}(r)=\left[1-\left(\frac{r_{+}}{r}\right)^{\tilde{d}}\right]^{-1}\left[1-\left(\frac{r_{-}}{r}\right)^{\tilde{d}}\right]^{\tilde{\gamma}_{\Omega}-1}, \\
& \tilde{C}(r)=\left[1-\left(\frac{r_{-}}{r}\right)^{\tilde{d}}\right]^{\tilde{\gamma}_{\Omega}}, \\
& \tilde{D}(r)=\left[1-\left(\frac{r_{-}}{r}\right)^{\tilde{d}}\right]^{\tilde{\gamma}_{x}},
\end{aligned}
$$


and

$$
\tilde{\gamma}_{x}=\frac{\tilde{d}}{d+\tilde{d}}, \quad \tilde{\gamma}_{\Omega}=\frac{\alpha^{2}(d)}{2 \tilde{d}}, \quad \tilde{\gamma}_{\phi}=-\alpha(d) .
$$

The "electric" charge $Q$ per unit volume of the black $(d-1)$-branes can be expressed in terms of $r_{-}$and $r_{+}\left(\geq r_{-}\right)$

$$
Q=\frac{\Omega_{\tilde{d}+1}}{4 \pi} \tilde{d}\left(r_{+} r_{-}\right)^{\tilde{d} / 2}
$$

where $\Omega_{\tilde{d}+1}$ is the volume of the unit $(\tilde{d}+1)$-sphere. One of the main goals of this work is to investigate the critical behavior in the black p-brane solutions with "electric" charge.

\section{B. Thermodynamics}

In order to investigate the critical behavior of black strings and $p$-branes, some relevant thermodynamic quantities must be derived first. The thermodynamics of these black configurations has been discussed by us and Muto [26], but a few misprints and calculation mistakes exist in [27]. For the completeness, here we list some main results of thermodynamics of these objects. Analytically continuing the solution (20) to its Euclidean sector leads to

$$
d s_{\mathrm{E}}^{2}=\tilde{A}(r) d \tau^{2}+\tilde{B}(r) d r^{2}+r^{2} \tilde{C}(r) d \Omega_{\tilde{d}+1}^{2}+\tilde{D}(r) d x^{j} d x^{j}
$$

where $\tau$ is the Euclidean time. We can obtain the Hawking temperature $T$ by requiring the absence of the conical singularity in the Euclidean spacetime

$$
T \equiv \beta_{H}^{-1}=\left.\frac{\tilde{A}^{\prime}}{4 \pi \sqrt{\tilde{A} \tilde{B}}}\right|_{r_{+}}=\frac{\tilde{d}}{4 \pi r_{+}}\left[1-\left(\frac{r_{-}}{r_{+}}\right)^{\tilde{d}}\right]^{(\tilde{d}-2) / 2 \tilde{d}}
$$

where a prime represents derivative with respect to $r$. The Hawking temperature (23) can also be derived from the surface gravity $\kappa(T=\kappa / 2 \pi)$ at the event horizon $r_{+}$, which satisfies

$$
l_{; \nu}^{\mu} l^{\nu}=\kappa l^{\mu}
$$

Here $l^{\mu}$ is a timelike Killing vector of solution (20). From (14) the Euclidean action of black $(d-1)$-brane is 


$$
\tilde{I}_{\mathrm{DE}}=-\frac{1}{16 \pi} \int d^{D} x \sqrt{g}\left(R-\frac{1}{2}(\nabla \phi)^{2}-\frac{e^{\alpha \phi}}{2(\tilde{d}+1) !} \tilde{F}_{\tilde{d}+1}^{2}\right)+\frac{1}{8 \pi} \int d^{D-1} x \sqrt{h}\left(K-K_{o}\right),
$$

where $K_{o}$ is the extrinsic curvature of a fixed $r>r_{+}$timelike supersurface embedded into the flat D-dimensional Minkovski spacetime. We have to introduce this term in order to normalize the Euclidean action to zero for a flat Minkovski spacetime [28]. For the Euclidean spacetime (22), we have

$$
\begin{array}{r}
R=-\frac{1}{\sqrt{g}}\left(\frac{\sqrt{g} \tilde{A}^{\prime}}{\tilde{A} \tilde{B}}\right)^{\prime}-2 G_{0}{ }^{0}, \\
K=-\frac{1}{\sqrt{g}}\left(\frac{\sqrt{g}}{\sqrt{\tilde{B}}}\right)^{\prime}, \quad K_{o}=-\frac{\tilde{d}+1}{r},
\end{array}
$$

where $G_{0}{ }^{0}$ is the 00-component of the Einstein tensor. Substituting (26) into (25) leads to the Euclidean action per unit $(d-1)$-brane volume

$$
\tilde{I}_{\mathrm{DE}}=\frac{\Omega_{\tilde{d}+1}}{16 \pi}\left[(\tilde{d}+1) r_{+}^{\tilde{d}}-r_{-}^{\tilde{d}}\right] \beta_{H}-\frac{1}{4} \Sigma-\frac{\pi Q}{\tilde{d} \Omega_{\tilde{d}+1} r_{+}^{\tilde{d}}} Q \beta_{H}
$$

where $\Sigma$ is the horizon area of the black $(d-1)$-brane,

$$
\Sigma=\Omega_{\tilde{d}+1} r_{+}^{\tilde{d}+1}\left[1-\left(\frac{r_{-}}{r_{+}}\right)^{\tilde{d}}\right]^{(\tilde{d}+2) / 2 \tilde{d}}
$$

Comparing the action (27) with the thermodynamic potential of an ordinary thermodynamic system [29], we obtain the Arnowitt-Deser-Misner (ADM) mass $M$, entropy $S$, and the chemical potential $\Phi$ with charge $Q$,

$$
\begin{aligned}
M & =\frac{\Omega_{\tilde{d}+1}}{16 \pi}\left[(\tilde{d}+1) r_{+}^{\tilde{d}}-r_{-}^{\tilde{d}}\right], \\
S & =\frac{1}{4} \Sigma=\frac{1}{4} \Omega_{\tilde{d}+1} r_{+}^{\tilde{d}+1}\left[1-\left(\frac{r_{-}}{r_{+}}\right)^{\tilde{d}}\right]^{(\tilde{d}+2) / 2 \tilde{d}}, \\
\Phi & =\frac{\pi}{\tilde{d} \Omega_{\tilde{d}+1}} \frac{Q}{r_{+}^{\tilde{d}}}=\frac{1}{4}\left(\frac{r_{-}}{r_{+}}\right)^{\tilde{d} / 2} .
\end{aligned}
$$

We note that the chemical potential $\Phi$ is just the electric potential at the horizon $r_{+}$. The ADM mass formula (29) was also obtained in [24] by using the gravitational energymomentum pseudotensor. Varying the ADM mass, one can obtain the first law of thermodynamics for the black extended objects 


$$
d M=\beta_{H}^{-1} d S+\Phi d Q,
$$

and its integration form is

$$
M=\frac{\tilde{d}+1}{\tilde{d}} \frac{\kappa}{2 \pi} \frac{\Sigma}{4}+\Phi Q .
$$

According to the formula $C_{Q}=(\partial M / \partial T)_{Q}$, the heat capacity is calculated as

$$
C_{Q}=\frac{r_{+} \Omega_{\tilde{d}+1}\left[(\tilde{d}+1) r_{+}^{\tilde{d}}+r_{-}^{\tilde{d}}\right]\left[1-\left(r_{-} / r_{+}\right)^{\tilde{d}}\right]^{(2+\tilde{d}) / 2 \tilde{d}}}{4\left[(\tilde{d}-1) r_{-}^{\tilde{d}} / r_{+}^{\tilde{d}}-1\right]} .
$$

Now let us discuss the thermodynamic properties of these black configurations. At the extremal limit $\left(r_{-}=r_{+}\right)$the Hawking temperature is zero, finite, and divergent when $\tilde{d}>2$, $\tilde{d}=2$, and $\tilde{d}<2$ (i.e., $\tilde{d}=1$ ), respectively. But the entropy always vanishes. The heat capacity (34) approaches zero in the extremal limit for a general $\tilde{d}$, whereas it is finite when $\tilde{d}=2$. We notice that the heat capacity is always negative for $\tilde{d}=1$. When

$$
(\tilde{d}-1) r_{-}^{\tilde{d}}-r_{+}^{\tilde{d}}=0,
$$

the heat capacity diverges. If $(\tilde{d}-1) r_{-}^{\tilde{d}}>r_{+}^{\tilde{d}}$, the heat capacity is positive and otherwise it is negative. Therefore, the points satisfying equation (35) correspond to the critical points of Davies. The point of Davies does not exist in the $p$-branes when $\tilde{d}=1$ and $\tilde{d}=2$. In addition, it is worth noting that the ADM mass $M$, "electric" charge $Q$, entropy $S$, and heat capacity $C_{Q}$ are the extensive quantities with respect to the volume of $(d-1)$-branes. Thanks to this, the entropy of near-extremal non-dilatonic $p$-branes may be described by free massless fields on the world volume [23]. It should be pointed out that these quantities only involve the dimensionality " $\tilde{d}$ ", but not the dimensionality " $d$ ". Finally, it is noted that the thermodynamics of the "magnetically" charged black $(\tilde{d}-1)$-branes can be obtained from the "electrically" charged black $(d-1)$-branes by the transformation $\tilde{d} \rightarrow d$. Hence their properties are similar to those of the "electrically" charged black $p$-branes.

\section{EQUILIBRIUM THERMODYNAMIC FLUCTUATIONS AND CRITICAL BEHAVIOR OF BLACK P-BRANES}




\section{A. Fluctuation theory of equilibrium thermodynamics}

In order to discuss the equilibrium thermodynamic fluctuations of black strings and $p$ branes, we first briefly review equilibrium fluctuation theory in specified environments. This was employed to study the critical behavior of Kerr-Newman black holes and BTZ black holes. In a self-gravitating thermodynamic system, the thermodynamic fluctuations are different in accordance with the different environments. Also thermodynamic stability depends on the chosen environment [30]. Hence, we must study the thermodynamic fluctuations of black configurations in various ensembles.

In general, the equilibrium state of a thermodynamic system can be described completely by the Massieu function $\Psi$ (the characteristic thermodynamic function). Let us consider the infinitesimal variation of the characteristic function [17],

$$
d \Psi=\sum_{i=1}^{n} X_{i} d x_{i}
$$

where the $n$ is the number of the variables $x_{i} .\left\{x_{i}\right\}$ is a set of the intrinsic variables $x_{i}$ and are specified directly by the environments of thermodynamic system. $\left\{X_{i}\right\}$ is a set of variables

conjugate to the set $\left\{x_{i}\right\}$, and can be expressed as functions of their intrinsic variables $\left\{x_{i}\right\}$ :

$$
X_{i}=\left(\frac{\partial \Psi}{\partial x_{i}}\right)_{\bar{x}_{i}}
$$

Here the subscript $\bar{x}_{i}$ denotes a set of variables $\left(x_{1}, \cdots, x_{i-1}, x_{i+1}, \cdots, x_{n}\right)$ exclusive of $x_{i}$. Then the deviation from the equilibrium state can be described by the distribution function

$$
P\left(\xi_{1}, \cdots, \xi_{n}\right) d \xi_{1} \cdots d \xi_{n} \sim \exp \left[k_{B}^{-1}(\hat{\Psi}-\Psi)\right] d \xi_{1} \cdots d \xi_{n}
$$

where $k_{B}$ is the Boltzmann's constant. $\xi_{i} \equiv \delta X_{i}$ stands for the deviation of the $i$ th conjugate variable from its equilibrium value. And $\hat{\Psi}$ represents the Massieu function which is analytically continued to the nonequilibrium points near the equilibrium sequence in the phase space $\left(\left\{x_{i}\right\},\left\{X_{i}\right\}\right)$. Considering this up to second order, one has [17]

$$
P\left(\left\{\xi_{i}\right\}\right) \sim \exp \left[-\left(2 k_{B}\right)^{-1} \sum_{i=1}^{n} \lambda_{i} \xi_{i}^{2}\right]
$$


where $\lambda_{i}=\left(\partial x_{i} / \partial X_{i}\right)_{\bar{x}_{i}}=\left(\partial^{2} \Psi / \partial x_{i}^{2}\right)_{\bar{x}_{i}}^{-1}$ are the eigenvalues of the fluctuation modes $\delta X_{i}$. It follows from (38) that the average of each fluctuation mode is zero, but the second moments are given by

$$
\left\langle\delta X_{i} \delta X_{j}\right\rangle=\frac{k_{B}}{\lambda_{i}} \delta_{i j}=k_{B}\left(\frac{\partial^{2} \Psi}{\partial x_{i}^{2}}\right)_{\bar{x}_{i}} \delta_{i j} .
$$

From (39) we can see that the second moments diverge only if $\lambda_{i}=0$, i.e., $\left(\partial X_{i} / \partial x_{i}\right)_{\bar{x}_{i}}=$ $\pm \infty$. According to the turning point method of stability analysis developed by Katz [31], the change of stability occurs only at the turning points where the tangent of the curve changes its sign through an infinity (a vertical tangent) in the "conjugate diagrams", in which the conjugate variables $\left\{X_{i}\right\}$ are plotted against an intrinsic variable (other intrinsic variables are fixed). The Davies' points are just such turning points. The divergent behavior of some second moments at the Davies' points means only that the stability for Kerr-Newman black holes is changed [16]. However this has nothing to do with the second-order phase transitions. On the other hand the divergence of some second moments at the extremal point is related to the second-order phase transition. This means that the extremal point is different from the turning point $[17,19]$. Therefore in the rest of paper we will explore the behavior of black configurations near the extremal limit.

\section{B. Equilibrium fluctuations of black strings and $p$-branes}

Here we investigate the equilibrium thermodynamic fluctuations of black strings and p-branes in the various ensembles.

(i) Microcanonical ensemble. In this ensemble, the proper Massieu function of a thermodynamic system is just the entropy of the system. For the black strings and p-branes, their entropy is given by the equation (30) and its variation can be obtained from (32)

$$
d \Psi_{1}=d S=\beta_{H} d M-\beta_{H} \Phi d Q
$$

Comparing (40) with (36), we have two intrinsic variables $\left\{x_{i}\right\}=\{M, Q\}$ and the conjugate variables $\left\{X_{i}\right\}=\left\{\beta_{H},-\varphi\right\}$ with $\varphi=\beta_{H} \Phi$. The eigenvalues for $\beta_{H}$ and $\varphi$ are given by 


$$
\begin{aligned}
\lambda_{1 m} & =\left(\frac{\partial M}{\partial \beta_{H}}\right)_{Q}=-T^{2} C_{Q} \\
\lambda_{1 q} & =-\left(\frac{\partial Q}{\partial \varphi}\right)_{M}=-T K_{M}
\end{aligned}
$$

where $C_{Q}$ is the heat capacity given by (34) and

$$
\begin{aligned}
K_{M} & \equiv \beta_{H}\left(\frac{\partial Q}{\partial \varphi}\right)_{M}=\frac{\tilde{d} \Omega_{\tilde{d}+1}}{\pi}\left(\frac{r_{+}}{r_{-}}\right)^{\tilde{d}}\left[(\tilde{d}+1) r_{+}^{\tilde{d}}+r_{-}^{\tilde{d}}\right] \\
& \times\left\{\left[(\tilde{d}+1) r_{+}^{\tilde{d}}-r_{-}^{\tilde{d}}\right]+\left[(\tilde{d}-1)-\left(r_{-} / r_{+}\right)^{\tilde{d}}\right]\left[1-\left(r_{-} / r_{+}\right)^{\tilde{d}}\right]^{-1}\right\}^{-1},
\end{aligned}
$$

is the "electric capacitance" of the black $p$-branes. It follows from (39) that

$$
\begin{aligned}
\left\langle\delta \beta_{H} \delta \beta_{H}\right\rangle & =-k_{B} \frac{\beta_{H}^{2}}{C_{Q}} \\
\langle\delta \varphi \delta \varphi\rangle & =-k_{B} \frac{\beta_{H}}{K_{M}} \\
\langle\delta \Phi \delta \Phi\rangle & =-k_{B}\left(\frac{T}{K_{M}}+\frac{\Phi^{2}}{C_{Q}}\right), \\
\left\langle\delta \beta_{H} \delta \Phi\right\rangle & =k_{B} \frac{\beta_{H} \Phi}{C_{Q}}
\end{aligned}
$$

$\lambda_{1 m}$ and $\lambda_{1 q}$ in (41) and (42) approach zero as $r_{-} \rightarrow r_{+}$for $\tilde{d}>2$ and $\tilde{d}=1$. Hence all these second moments diverge in the extremal limit $\left(r_{-}=r_{+}\right)$. For $\tilde{d}=2$, all these second moments are finite even in the extremal limit. The divergence of second moments for $\tilde{d} \neq 2$ implies that the extremal limit is on a critical point. In addition, it should be pointed out that, in this ensemble, all second moments are finite at the points of Davies.

(ii) Canonical ensemble. In this case, the black $p$-branes can only exchange the heat with surroundings. The variation of proper Massieu function is

$$
d \Psi_{2}=d S-d\left(\beta_{H} M\right)=-M d \beta_{H}-\varphi d Q
$$

Here the intrinsic variables become $x_{i}=\left\{\beta_{H}, Q\right\}$, and the conjugate variables $X_{i}=$ $\{-M,-\varphi\}$. The eigenvalues are

$$
\begin{aligned}
\lambda_{2 \beta_{H}} & =-\left(\frac{\partial \beta_{H}}{\partial M}\right)_{Q}=\frac{\beta_{H}^{2}}{C_{Q}} \\
\lambda_{2 q} & =-\left(\frac{\partial Q}{\partial \varphi}\right)_{\beta_{H}}=-\frac{K_{\beta_{H}}}{\beta_{H}}
\end{aligned}
$$


where

$$
K_{\beta_{H}} \equiv \beta_{H}\left(\frac{\partial Q}{\partial \varphi}\right)_{\beta_{H}}=\frac{\tilde{d} \Omega_{\tilde{d}+1}\left(r_{+} r_{-}\right)^{\tilde{d} / 2}}{\pi} \frac{\left[1-(\tilde{d}-1)\left(r_{-} / r_{+}\right)^{\tilde{d}}\right]}{\left[1-\left(r_{-} / r_{+}\right)^{\tilde{d}}\right]}
$$

From (34), we obtain second moments as follows:

$$
\begin{aligned}
\langle\delta M \delta M\rangle & =k_{B} T^{2} C_{Q}, \\
\langle\delta S \delta M\rangle & =k_{B} T C_{Q}, \\
\langle\delta \varphi \delta \varphi\rangle & =-k_{B} \frac{\beta_{H}}{K_{\beta_{H}}}, \\
\langle\delta \Phi \delta \Phi\rangle & =-k_{B} \frac{T}{K_{\beta_{H}}}, \\
\langle\delta S \delta S\rangle & =k_{B} C_{Q} .
\end{aligned}
$$

For $\tilde{d}>2$ and $\tilde{d}=1$, the eigenvalues $\lambda_{2 \beta_{H}}$ and $\lambda_{2 q}$ diverges in the extremal limit. Thus all second moments are finite, and approach zero in the extremal limit. For $\tilde{d}=2$, all these second moments are finite even if the extremal limit is taken. We wish to comment that contrary to the microcanonical ensemble, all second moments diverge at the Davies' points in canonical ensemble.

(iii) Grand canonical ensemble. In this case the black p-branes can exchange the heat with surroundings. Also they can do work on the surroundings. The variation of the proper Massieu function is

$$
d \Psi_{3}=d \Psi_{2}+d(\varphi Q)=-M d \beta_{H}+Q d \varphi .
$$

Here we read the intrinsic variables $x_{i}=\left\{\beta_{H}, \varphi\right\}$, and the conjugate variables $X_{i}=$ $\{-M, Q\}$. The corresponding eigenvalues lead to

$$
\begin{gathered}
\lambda_{3 \beta_{H}}=-\left(\frac{\partial \beta_{H}}{\partial M}\right)_{\varphi}=\frac{\beta_{H}^{2}}{C_{\varphi}}, \\
\lambda_{3 \varphi}=\left(\frac{\partial \varphi}{\partial Q}\right)_{\beta_{H}}=\frac{\beta_{H}}{K_{\beta_{H}}},
\end{gathered}
$$

where 


$$
\begin{aligned}
C_{\varphi} & \equiv\left(\frac{\partial M}{\partial T}\right)_{\varphi}=\frac{\Omega_{\tilde{d}+1} r_{+}^{2}}{4}\left[1-\left(\frac{r_{-}}{r_{+}}\right)^{\tilde{d}}\right]^{(\tilde{d}+2) / 2 \tilde{d}}\left[(\tilde{d}+1) r_{+}^{\tilde{d}-1}-r_{-}^{\tilde{d}-1} \mathcal{G}\right] \\
& \times\left\{\left[-1+\frac{\tilde{d}}{2}\left(\frac{r_{-}}{r_{+}}\right)^{\tilde{d}}\right]-\frac{\tilde{d}-2}{2}\left(\frac{r_{-}}{r_{+}}\right)^{\tilde{d}-1} \mathcal{G}\right\}^{-1},
\end{aligned}
$$

and

$$
\mathcal{G}=\frac{\tilde{d}-2}{2}\left(\frac{r_{-}}{r_{+}}\right)\left[\frac{\tilde{d}}{2}-\left(\frac{r_{-}}{r_{+}}\right)^{\tilde{d}}\right]^{-1} .
$$

In this ensemble, the nonvanishing second moments are

$$
\begin{aligned}
\langle\delta M \delta M\rangle & =k_{B} T^{2} C_{\varphi}, \\
\langle\delta Q \delta Q\rangle & =k_{B} T K_{\beta_{H}} \\
\langle\delta S \delta S\rangle & =k_{B}\left(C_{\varphi}+\varphi^{2} T K_{\beta_{H}}\right), \\
\langle\delta S \delta M\rangle & =k_{B} T C_{\varphi} \\
\langle\delta Q \delta S\rangle & =-k_{B} \varphi^{2} T K_{\beta_{H}} .
\end{aligned}
$$

For $\tilde{d}>2$, we have $\lambda_{3 \beta_{H}} \rightarrow \infty$ and $\lambda_{3 \varphi} \rightarrow 0$ in the extremal limit. Thus, $\langle\delta M \delta M\rangle$ and $\langle\delta S \delta M\rangle$ are finite, while $\langle\delta Q \delta Q\rangle,\langle\delta Q \delta S\rangle$, and $\langle\delta S \delta S\rangle$ diverge under the extrtemal limit.

For $\tilde{d}=1,\langle\delta S \delta S\rangle$ and $\langle\delta Q \delta S\rangle$ diverge but others are finite in the extremal limit. For $\tilde{d}=2$, all these second moments are finite again. In this ensemble, all second moments are also finite at the points of Davies.

\section{Scaling laws and critical exponents}

Up to now we have calculated some second moments for different fluctuation modes. It is shown that the thermodynamic fluctuations and second moments of black $p$-branes are resulted in different, according to the different environments. In order to discuss the critical behavior of the isolated black $p$-branes as in $[18,19]$, it is appropriate to choose the microcanonical ensemble. Here the proper Massieu function is given by the entropy of black p-branes. From (44) it follows that all second moments diverge in the extremal limit. Therefore it is suggested that the extremal black $p$-branes has a critical point, as in 
the cases of the Kerr-Newman black holes and BTZ black holes. According to the ordinary thermodynamics, it is conjectured that the extremal and nonextremal black $p$-branes are two different phases. As is well known, the extremal black configurations are very different from the nonextremal black configurations in many respects. For example, there exists the difference of the topological structures between the extremal and nonextremal black holes $[32,33]$. In the Euclidean manifolds of black holes, a conical singularity exists at the event horizon for a nonextremal black hole and results in the periodicity of the Euclidean time in order to remove the singularity, which gives us the inverse Hawking temperature of the black hole. The periodicity is absent for extremal black holes. This gives rise to the argument of Hawking et al [32] that an extremal black hole can be in the equilibrium with an arbitary temperature heat bath. A nonextremal black hole can be described by the black hole thermodynamics. But the thermal description fails for some extremal black holes [15]. In addition, the mechanism of radiations is also different for the extremal and nonextremal black holes. There exist two kinds of radiations, the Hawking evaporation and the superradiation, in the nonextremal black holes. For an extremal black hole only the superradiation is left because the Hawking temperature is zero (although the Hawking temperature of some dilaton black holes becomes infinity under the extremal limit, the Hawking evaporation is still killed by an infinit gap outside the black holes [15]). In particular, it has been shown that some extremal black configurations are supersymmetric and the supersymmetry is absent for their nonextremal counterparts. Some shown examples are the Reissner-Nordström black holes [34], BTZ black holes [35], dilaton black holes [36], and black strings and p-branes [21, $22,37]$. Therefore, we argue that the extremal limit is a critical point and a second-order phase trasition takes place from the extremal to nonextremal black $p$-branes. The extremal and nonextremal black $p$-branes are in the different phases. The extremal black $p$-branes are in the disordered phase and the nonextremal in the ordered phase because the extremal black p-branes have higher symmetry than the nonextremal. Accompanying the phase transition is the breaking of the supersymmetry.

In the phase transition theory of ordinary thermodynamics, the order parameter is a very 
important physical quantity. In general, the order parameter is chosen among the extensive variables or their densities $[38,39]$. One of the extensive variables, for example, $a_{j}=X$ is related to the order parameter $\eta=X-X_{1}$, where $X_{1}$ is the equilibrium value of $X$ in the disordered phase. Thus, the order parameter is equal to zero and is defferent from zero in the ordered phase. In the ordinary thermodynamic system, the extensive variables are different and the intensive variables are common in the two phases. But the intensive quantities are different and the extensive ones are common in the two phases of black holes. For the nonextremal $p$-branes in microcanonical ensemble, the intensive quantities $\left(\beta_{H}, \varphi\right)$ are different, while the extensive ones $(M, Q)$ are same at the $r_{+}$and $r_{-}$. We can thus regard the differences $\eta_{\beta_{H}}=\beta_{H+}-\beta_{H-}$ and $\eta_{\varphi}=\varphi_{+}-\varphi_{-}$as the order parameters of the black p-branes. Here the suffixes "+" and "-" mean that the quantity is taken at the $r_{+}$and $r_{-}$, respectively. When the extremal limit is approached, one can observe the critical bebavior of order parameters. Differing from the order parameters in the usual thermodynamic systems, the order parameters of the $\tilde{d}>2$ black $p$-branes diverge under the extremal limit. This is because the critical temperature is zero in this phase transition. On the other hand, the second-order derivatives of entropy with respect to the intrinsic variables are given by the inverse eigenvalues

$$
\begin{gathered}
\bar{\zeta}_{1} \equiv\left(\frac{\partial^{2} S}{\partial M^{2}}\right)_{Q}=\lambda_{1 m}^{-1}=-\frac{\beta_{H}^{2}}{C_{Q}} \\
\bar{\zeta}_{2} \equiv\left(\frac{\partial^{2} S}{\partial Q^{2}}\right)_{M}=\lambda_{1 q}^{-1}=-\frac{\beta_{H}}{K_{M}} .
\end{gathered}
$$

According to Kaburaki [18], the critical exponents of these quantities can be given as follows,

$$
\begin{aligned}
\bar{\zeta}_{1} & \sim \varepsilon_{M}^{-\alpha} \quad(\text { for } \mathrm{Q} \text { fixed }) \\
& \sim \varepsilon_{Q}^{-\psi} \quad(\text { for } \mathrm{M} \text { fixed }) \\
\bar{\zeta}_{2} & \sim \varepsilon_{M}^{-\gamma} \quad(\text { for } \mathrm{Q} \text { fixed }) \\
& \sim \varepsilon_{Q}^{-\sigma} \quad(\text { for } \mathrm{M} \text { fixed }) \\
\eta_{\varphi} & \sim \varepsilon_{M}^{\beta} \quad(\text { for } \mathrm{Q} \text { fixed }), \\
& \left.\sim \varepsilon_{Q}^{\delta^{-1}} \quad \text { (for } \mathrm{M} \text { fixed }\right)
\end{aligned}
$$


where $\varepsilon_{M}$ and $\varepsilon_{Q}$ represent the infinitesimal deviations of $M$ and $Q$ from their limit values. Making use of Eqs.(55) and (56), we obtain

$$
\alpha=\psi=\gamma=\sigma=(3 \tilde{d}-2) / 2 \tilde{d}, \quad \beta=\delta^{-1}=-(\tilde{d}-2) / 2 \tilde{d}
$$

The critical exponents $\beta$ and $\delta^{-1}$ are negative for $\tilde{d}>2$, which shows that in this case the order parameter $\eta_{\varphi}$ diverges in the extremal limit $\left(r_{-} \rightarrow r_{+}\right)$. We note that these exponents are different from those of Kerr-Newman black holes and BTZ black holes [19]. But they still satisfy the scaling laws of the "first kind" as

$$
\begin{aligned}
& \alpha+2 \beta+\gamma=2, \\
& \beta(\delta-1)=\gamma \\
& \psi(\beta+\gamma)=\alpha .
\end{aligned}
$$

That the scaling laws (61) hold in the black $p$-branes is related to the fact that the black $(d-1)$-brane entropy is a homogeneous function, satisfying

$$
S(\lambda M, \lambda Q)=\lambda^{(\tilde{d}+1) / \tilde{d}} S(M, Q),
$$

where $\lambda$ a positive constant. This can be seen clearly from Eqs. (21), (29) and (30).

In the ordinary thermodynamic system, the correlation function is an important quantity to extract the information for phase transitions. Near the critical points, this function takes generally the form $[38,39]$

$$
G(r) \sim \frac{\exp (-r / \xi)}{r^{\bar{d}-2+\eta}}
$$

where $\eta$ is the Fisher's exponent, $\bar{d}$ is the effective spatial dimension of the system under consideration, and $\xi$ is the correlation length. Applying this to our system, we have

$$
\begin{aligned}
& \xi \sim \varepsilon_{M}^{-\nu} \quad(\text { for } Q \text { fixed }) \\
& \sim \varepsilon_{Q}^{-\mu} \quad(\text { for } M \text { fixed })
\end{aligned}
$$

Further these critical exponents satisfy the scaling laws of the "second kind", 


$$
\nu(2-\eta)=\gamma, \quad \nu \bar{d}=2-\alpha, \quad \mu(\beta+\gamma)=\nu
$$

Due to the absence of quantum theory of gravity, by now the correlation function of quantum black holes has yet been unclear. Instead, we use the correlation function of scalar fields to get the quantum aspects of black p-branes [19]. Traschen [40] has recently studied the behavior of a massive charged scalar field on the background of Reissner-Nordström black holes. Traschen has found that the spacetime geometry near the horizon of the extremal Reissner-Nordström black holes has a scaling symmetry, which is absent for the nonextremal holes, a scale being introduced by the surface gravity. The scaling symmetry results in that an external source has a long range influence on the extremal background as $y^{-1}$, compared to a correlation length scale which falls off exponentially fast in the case of nonextremal holes, like $e^{2 \kappa y}$, where $\kappa$ is the surface gravity and $y$ is the usual tortoise coordinate. Therefore the inverse surface gravity is regarded as the correlation length. Here we assume that this also holds for our black $p$-branes. Making use of the surface gravity of black $p$-branes, we obtain

$$
\nu=\mu=(\tilde{d}-2) / 2 \tilde{d} .
$$

Substituting these into (65), we have

$$
\eta=-(\tilde{d}+2) /(\tilde{d}-2), \quad \bar{d}=(\tilde{d}+2) /(\tilde{d}-2) .
$$

The two critical exponents are also different from the BTZ black holes [19]. In particular, for $\tilde{d}=1$, the effective dimension $\bar{d}=-3$. The appearance of the negative effective dimensionality is due to that the surface gravity of $\tilde{d}=1$ black $p$-branes is divergent under the extremal limit. More recently, such a negative exponent scaling has also been found by Klebanov and Tseytlin [41] in the intersecting M-brane configurations. They argued that this scaling can be explained by the dynamical $p$-branes. In addition, unlike in the BTZ black holes, in which the effective dimension $\bar{d}=1$, from (67) we see that generally the effective dimension of these dilaton $p$-branes is not an integer.

So far, we have investigated the critical behavior of black $(d-1)$-branes with "electric" charge. The extremal limit of $(d-1)$-branes has a critical point and a second-order phase 
transition may take place from the extremal to nonextremal black $p$-branes. It is already shown that these extremal black $p$-branes are supersymmetric and the supersymmetry is always absent for their nonextremal counterparts [21, 22, 37]. Therefore, that a phase transition occurs from extremal to nonextremal black $p$-branes agrees with the fact of symmetric changes. We have argued that the extremal black $p$-branes and nonextremal black $p$-branes are two different phases, and the extremal black $p$-branes are in the disordered phase and the nonextremal black $p$-branes in the ordered phase. However $\tilde{d}=2$ is a special case. In this case, the extremal limit does not have a critical point because the thermodynamic quantities and fluctuations have good bahavior even in the extremal limit. This situation is similar to that of the $a=1$ charged dilaton black holes. However, if one considers other fields and/or quantum corrections in the theory, we argue that the extremal limit will have a critical point. This will be seen below.

\section{CHARGED DILATON BLACK HOLES}

It is well known that the behavior of black holes is drastically changed because of the presence of dilaton fields. In order to understand the effect of the dilaton field on the critical behavior of black holes, we choose the four dimensional dilaton action [42],

$$
S=\frac{1}{16 \pi} \int d^{4} x \sqrt{-g}\left[R-2(\nabla \phi)^{2}-e^{-2 a \phi} F^{2}\right]
$$

where $F$ is the Maxwell field. The coupling constant $a$ governs the interaction between the dilaton field and the Maxwell field. For $a=1$, the action corresponds to the low-energy approximation of superstring theory. The charged black hole solutions are

$$
\begin{aligned}
& F=\frac{e^{2 a \phi}}{R^{2}} Q, \\
& e^{2 a \phi}=\left(1-\frac{r_{-}}{r_{+}}\right)^{2 a^{2} /\left(1+a^{2}\right)}, \\
& d s^{2}=-A^{2}(r) d t^{2}+A^{-2}(r) d r^{2}+R^{2}(r) d \Omega^{2},
\end{aligned}
$$

where 


$$
\begin{aligned}
& A^{2}(r)=\left(1-\frac{r_{+}}{r}\right)\left(1-\frac{r_{-}}{r}\right)^{\left(1-a^{2}\right) /\left(1+a^{2}\right)} \\
& R^{2}(r)=r^{2}\left(1-\frac{r_{-}}{r}\right)^{2 a^{2} /\left(1+a^{2}\right)}
\end{aligned}
$$

Two parameters $r_{+}$and $r_{-}$are related to the mass $M$ and electric charge $Q$,

$$
2 M=r_{+}+\frac{1-a^{2}}{1+a^{2}} r_{-}, \quad Q^{2}=\frac{r_{-} r_{+}}{1+a^{2}} .
$$

The Hawking temperature $T$ and entropy $S$ of the dilaton black holes are

$$
\begin{aligned}
& T=\frac{1}{4 \pi r_{+}}\left(1-\frac{r_{-}}{r_{+}}\right)^{\left(1-a^{2}\right) /\left(1+a^{2}\right)}, \\
& S=\pi r_{+}^{2}\left(1-\frac{r_{-}}{r_{+}}\right)^{2 a^{2} /\left(1+a^{2}\right)} .
\end{aligned}
$$

The heat capacity of the dilaton black holes $\left(C_{Q}=(\partial M / \partial T)_{Q}\right)$ is found to be

$$
C_{Q}=-\frac{2 \pi r_{+}^{2}\left[1-\frac{1-a^{2}}{1+a^{2}} \frac{r_{-}}{r_{+}}\right]\left[1-\frac{r_{-}}{r_{+}}\right]^{2 a^{2} /\left(1+a^{2}\right)}}{\left[1-\frac{3-a^{2}}{1+a^{2}} \frac{r_{-}}{r_{+}}\right]}
$$

From Eqs.(72)-(74) we easily find that the thermodynamic quantities depend explicitly on the coupling constant $a$. In the extremal limit $\left(r_{-} \rightarrow r_{+}\right)$, the Hawking temperature is zero, finite, and divergent when $a<1, a=1$, and $a>1$, respectively. But the entropy always vanishes. For $a>1$, the heat capacity is always negative and approaches zero in the extremal limit. When $a=1$, the heat capacity $C_{Q}=-8 \pi M^{2}$, independent of the electric charge $Q$. Furthermore, for $a<1$, it is possible to have

$$
1-\frac{3-a^{2}}{1+a^{2}} \frac{r_{-}}{r_{+}}=0
$$

which means that $C_{Q}$ diverges in this case. These points correspond to the turning points of Davies. At these points, the heat capacity encounters with an infinite discontinuity. With (71)-(73), we have the first law of thermodynamics

$$
d M=\beta_{H}^{-1} d S+\Phi d Q
$$

where $\beta_{H}^{-1}=T$ and $\Phi=Q / r_{+}$is the electric potential at the horizon $r_{+}$. The mass formula of the holes is 


$$
M=2 \frac{\kappa}{2 \pi} \frac{\Sigma}{4}+\Phi Q
$$

where $\kappa(\Sigma)$ are the surface gravity (the horizon area) of the black holes.

Applying the results for the $p$-branes in the microcanonical ensemble to this system, the eigenvalues and nonvanishing second moments are still given by Eqs. (41), (42) and (44), respectively. But the heat capacity $C_{Q}$ should be replaced by the expression (74), and $K_{M}$ is

$$
K_{M}=\left[\frac{1+a^{2}}{1-a^{2}}-\frac{r_{-}}{r_{+}}\right]\left[\frac{1}{r_{+}}\left(\frac{1+a^{2}}{1-a^{2}}+\frac{r_{-}}{r_{+}}\right)+\frac{4 r_{-}^{2}}{\left(1+a^{2}\right) r_{+}^{3}}\left(1-\frac{r_{-}}{r_{+}}\right)^{-1}\right]^{-1} .
$$

Again we find that two eigenvalues of $\beta_{H}$ and $\varphi$ approach zero in the extremal limit, and hence all these second moments (44) diverge. For the case of $a=1$, the eigenvalues and second moments are always finite. Therefore the all extremal black holes except $a=1$ have critical points. Similarly, we can obtain relevant critical exponents

$$
\alpha=\psi=\gamma=\sigma=\frac{2}{1+a^{2}}, \quad \beta=\delta^{-1}=-\frac{1-a^{2}}{1+a^{2}}
$$

Naturally, these also satisfy the scaling laws of the "first kind" (61), because the entropy (73) is a homogeneous function, which satisfies

$$
S(\lambda M, \lambda Q)=\lambda^{2} S(M, Q)
$$

with a positive constant $\lambda$. From the surface gravity and the scaling laws of the "second kind", we have

$$
\nu=\mu=\frac{1-a^{2}}{1+a^{2}}
$$

and

$$
\eta=-\frac{2 a^{2}}{1-a^{2}}, \quad \bar{d}=\frac{2 a^{2}}{1-a^{2}} .
$$

Here the negative effective dimension $\bar{d}$ appears again when $a>1$. This arises from the same reason as in the $(d-1)$-branes with $\tilde{d}=1$, because the surface gravity diverges in the 
extremal limit. For $a=0$, the charged dilaton black holes reduce to the Reissner-Nordström black holes. After some calculations, we have

$$
\begin{aligned}
& \alpha=\psi=\gamma=\sigma=3 / 2, \quad \beta=\delta^{-1}=-1 / 2, \\
& \nu=\mu=1 / 2, \quad \eta=-1, \quad \bar{d}=1 .
\end{aligned}
$$

These exponents are exactly the same as those of the BTZ black holes [19]. The critical behavior of the extremal dilaton black holes in the microcanonical ensemble is in complete agreement with the result of nonequilibrium thermodynamic fluctuations [14]. The thermodynamic fluctuations of dilaton black holes in the canonical ensemble and grand canonical ensemble can also be given similarly as in the black $p$-branes. In the canonical ensemble, the divergent point of second moments is the point of Davies (75).

From the above, we find that the extremal black $p$-branes and dilaton black holes have critical points, and the relevant critical exponents satisfy the scaling laws. This is the important aspect of phase transitions. On the other hand, the extremal $\tilde{d}=2$-branes and extremal $a=1$ dilaton black holes have not the critical points and show no features of phase transitions. In addition, the $\tilde{d}=1$ black branes and $a>1$ dilaton black holes have the negative effective spatial dimension. Concerning this problem, although Klebanov and Tseytlin [41] have made some discussions in the intersecting M-brane configurations, to understand completely the negative scaling in all appearing cases, further investigation is explicitly needed. For example, we need to investigate further whether or not these extremal black configurations have the scaling symmetry and the external source has the long range influence on these extremal black configuration backgrounds. For the $\tilde{d}=2$ black branes and $a=1$ dilaton black holes, we would like to point out that if one further considers other fields in the string theories, the extremal limit should be a critical point, because the actions (1) and (68) are the simpler approximations of the lower-energy actions of string theories. Recall that the action (68) with parameter $a=1$ is just the lower-energy limit of the heterotic superstring theory, in order to study the relationship between extremal black configurations and strings, it is necessary to consider the dilaton black holes with other fields 
and/or quantum corrections. In the next section, we will study the $a=1$ charged dilaton black hole with a moduli field.

\section{CHARGED DILATON BLACK HOLES WITH MODULI FIELDS}

The actions (1) and (68) are the first approximations of lower-energy actions of string theories. For the large mass black holes $\left(M>>M_{\mathrm{pl}}\right)$, these should be a good approximation. But, here we are considering the quantum effects of these configurations, in order to understand the critical behavior of these black configurations in the string theories, the

effects of other fields in the string theories and/or quantum corrections to the lower-energy action of string theories should be investigated. In this section, we study the charged dilaton black holes with moduli fields. These come from the low-energy action of heterotic theories by compactification from ten to four dimensions. Here we include one single modulus which describes the radius of a compactified space. The action is

$$
S=\frac{1}{16 \pi} \int d^{4} x \sqrt{-g}\left[R-2(\nabla \phi)^{2}-\frac{2}{3}(\nabla \sigma)^{2}-e^{-2 \phi} F^{2}-e^{-2 q \sigma / 3} F^{2}\right] .
$$

This is a modification of the action (68) with $a=1$ by including a moduli field $\sigma$ with a coupling constant $q$. The black hole solutions in the action (84) are found already by Cadoni and Mignemi [43]. The metric takes the form in Eq. (69) but with the substitutions

$$
\begin{aligned}
& A^{2}(r)=\left[1-\frac{r_{+}}{r}\right]\left[1-\frac{r_{-}}{r}\right]^{3 /\left(2 q^{2}+3\right)}, \\
& R^{2}(r)=r^{2}\left[1-\frac{r_{-}}{r}\right]^{2 q^{2} /\left(2 q^{2}+3\right)}, \\
& e^{-2 \phi}=\left[1-\frac{r_{-}}{r}\right]^{2 q^{2} /\left(2 q^{2}+3\right)}, \\
& F=Q \varepsilon_{2}, \quad \sigma=3 \phi / q .
\end{aligned}
$$

This is a two-parameter black hole solution with magnetic charge $Q$. The electrically charged solutions can be obtained by the duality transformation. The ADM mass $M$ and charge $Q$ have the relations with two parameters $r_{-}$and $r_{+}$, 


$$
\begin{aligned}
& 2 M=r_{+}+\frac{3}{2 q^{2}+3} r_{-}, \\
& Q^{2}=\frac{q^{2}+3}{2 q^{2}+3} r_{-} r_{+} .
\end{aligned}
$$

The Hawking temperature and entropy of the black holes are easily calculated as

$$
\begin{aligned}
& T=\frac{1}{4 \pi r_{+}}\left[1-\frac{r_{-}}{r_{+}}\right]^{3 /\left(2 q^{2}+3\right)}, \\
& S=\pi r_{+}^{2}\left[1-\frac{r_{-}}{r_{+}}\right]^{2 q^{2} /\left(2 q^{2}+3\right)} .
\end{aligned}
$$

The heat capacity is

$$
C_{Q}=-2 \pi r_{+}^{2}\left[1-\frac{r_{-}}{r_{+}}\right]^{2 q^{2} /\left(2 q^{2}+3\right)}\left[1-\frac{3}{2 q^{2}+3} \frac{r_{-}}{r_{+}}\right]\left[1-\frac{2 q^{2}+9}{2 q^{2}+3} \frac{r_{-}}{r_{+}}\right]^{-1} .
$$

From Eqs. (88)-(90), it is clear that the thermodynamics of the $a=1$ charged dilaton black holes is changed drastically due to the presence of the moduli field. In this case, the Hawking temperature and entropy are zero in the extremal limit $\left(r_{-} \rightarrow r_{+}\right)$. This means that the extremal limit corresponds to a stable nondegenerate ground state. In addition, the entropy (89) is still a homogeneous function of the form (80). When one goes to the extremal limit, the heat capacity (90) approaches zero. But this diverges and changes its sign, when goes to points of Davies which satisfy the relation

$$
1-\frac{2 q^{2}+9}{2 q^{2}+3} \frac{r_{-}}{r_{+}}=0
$$

Now we are in a position to consider the equilibrium thermodynamic fluctuations of this model in the microcanonical ensemble. These eigenvalues and second moments are still given by Eqs. (41), (42) and (44), respectively. In this case, $C_{Q}$ is given by Eq. (90) and $K_{M}$ is

$$
K_{M}=\left[\frac{2 q^{2}+3}{3}-\frac{r_{-}}{r_{+}}\right]\left\{\frac{1}{r_{+}}\left[\frac{2 q^{2}+3}{3}+\frac{r_{-}}{r_{+}}\right]+\frac{2 q^{2}+6}{2 q^{2}+3} \frac{r_{-}^{2}}{r_{+}^{3}}\left[1-\frac{r_{-}}{r_{+}}\right]^{-1}\right\}^{-1} .
$$

The eigenvalues of the fluctuation modes become zero and all second moments diverge at the extremal limit. Similarly, corresponding critical exponents are easily obtained and read as 


$$
\alpha=\psi=\gamma=\sigma=\frac{2 q^{2}+6}{2 q^{2}+3}, \quad \beta=\delta^{-1}=-\frac{3}{2 q^{2}+3} .
$$

The critical exponents of the correlation length are

$$
\nu=\mu=\frac{3}{2 q^{2}+3}
$$

and from the scaling laws of the "second kind", we have

$$
\eta=-2 q^{2} / 3, \quad \bar{d}=2 q^{2} / 3
$$

Thus this model has a critical point because of the presence of the moduli fields. We argue that the extremal black configuration in the superstring theories has the critical point, and related exponents satisfy the scaling laws. In the canonical ensemble, all second moments are finite except the points of Davies (91). It is worth noting that when $q=0$, the solution (85) does not reduce to the $a=1$ dilaton black holes, but the Reissner-Nordström black hole. In the string frame $\left(d s_{S}^{2}=e^{2 \phi} d s^{2}\right)$, the metric (85) becomes

$$
d s_{S}^{2}=-\left[1-\frac{r_{+}}{r}\right]^{6 /\left(2 q^{2}+3\right)} d t^{2}+\left[1-\frac{r_{+}}{r}\right]^{-2} d r^{2}+r^{2} d \Omega^{2}
$$

in the extremal limit. The geometry of the $t=$ constant surface is exactly identical to that of a static slice in the extremal Reissner-Nordström spacetime. Here is a semi-infinite throat attached to an asymptotically flat region. This supports that the extremal dilaton black holes with moduli field has a critical point, as in the case of the Reissner-Nordström black holes.

\section{CONCLUSION AND DISCUSSION}

In this work we investigated the critical behavior for the black $p$-branes and four dimensional dilaton black holes with (and without) a moduli field. We confirmed that the thermodynamic fluctuations depend on the kind of ensembles. Hence three ensembles for a self-gravitating system are not equivalent. In the microcanonical ensemble, all second moments of extremal black configurations diverge. Some second moments diverge at the points 
of Davies in the canonical ensemble. But the points of Davies are actually the turning points, which reveal to the changes of stability and have nothing to do with phase transition. The extremal black configurations has a critical point. And the phase transition takes place from the extremal to nonextremal black configurations. The related critical exponents satisfying the scaling laws are obtained. Note that the entropy of these black configurations takes a property of the homogeneous function.

A phase transition is always accompanied by the symmetry changes in the ordinary thermodynamic systems. As is well known, some extremal black configurations are supersymmetric and the supersymmetry is absent for nonextremal ones. Thus it is suggested that the phase transition from the extremal to nonextremal black configurations takes the change of supersymmetry. We argued that the extremal and nonextremal black configurations are two different phases. The extremal black configurations are in the disordered phase and the nonextremal in the ordered phase because the extremal black configurations have higher symmetry than the nonextremal ones. For the $\tilde{d}=1$ black $p$-branes and $a>1$ charged dilaton black holes, the effective spatial dimension is negative. This is because of the divergence of surface gravity for these extremal black configurations. In order to understand the origin of the negative scaling, further study beyond the scope of this paper should be required. The recent work of Klebanov and Tseytlin [41] seems to point out a promising direction. In addition, to further investigate the critical behavior of these special extremal configurations, it is of interest to study that the behavior of quantum fields on the these extremal configuration backgrounds, as was done by Traschen [40] on the Reissner-Nordström background, to see whether or not the geometry of these extremal configurations has the scaling symmetry, and whether the external sources have the long range influence on these extremal backgrounds. The extremal $\tilde{d}=2$ black branes and $a=1$ dilaton black holes do not have a critical point. It is, however, found a critical point for the extremal limit by adding a moduli field to the $a=1$ dilaton black hole. Finally, we notice that the microscopic understanding of entropy for the black holes and black $p$-branes need the condition of the contant dilaton field [23]. And we wish to recall that the Reissner-Nordström and BTZ black holes can be considered 
as the exact solutions in the string theories with constant dilaton fields $[42,43,44]$. In this sense it is very important to investigate the critical behavior of black configurations with constant dilaton fields 円.

\section{Acknowledgments}

R. G. Cai wishes to thank O. Kaburaki for sending some recent works to him, Dr. Y. K. Lau for useful discussions, and Professor J. D. Bekenstein for helpful comments on the cirtical behavior in BTZ black holes [19]. We would like to thank the referee for many helpful suggestions which improved the original version of this paper. The research of R.G.C. was supported in part by China Postdoctoral Science Foundation.

\footnotetext{
${ }^{1}$ After completing this work, we studied further the scaling laws and critical exponents of the non-dilatonic black $p$-branes [37]. It was found that the effective spatial dimension is one for non-dilatonic black holes and black strings, and is just $p$ for the non-dilatonic $p$-branes [45]. This result can explain why the Bekenstein-Hawking entropy may be given a simple world volume interpretation only for the non-dilatonic $p$-branes.
} 


\section{REFERENCES}

[1] S. W. Hawking, Nature 248 (1974) 30; Commun. Math. Phys. 43 (1975) 199.

[2] J. M. Bardeen, B. Carter, and S. W. Hawking, Commun. Math. Phys. 31 (1973) 161.

[3] J. D. Bekenstein, Phys. Rev. D 7 (1973) 2333; D 9 (1974) 3294.

[4] R. M. Wald, General Relativity (Chicago University Press, Chicago, 1984).

[5] P. C. W. Davies, Proc. R. Soc. London A 353 (1977) 499; Class. Quantum Grav. 6 (1989) 1909.

[6] P. Hut, Mon. Not. R. Astron. Soc. 180 (1977) 379.

[7] L. M. Sokolowski and P. Mazur, J. Phys. A 13 (1980) 1113.

[8] Y. K. Lau, Phys. Lett. A186 (1994) 41; A 188 (1994) 245.

[9] C. O. Lousto, Nucl. Phys. B 410 (1993) 155, Erratum B 449 (1995) 433; Gel. Rel. Grav. 27 (1995) 121; Phys. Rev. D 51 (1995) 1733.

[10] J. P. Muniain and D. Píriz, Phys. Rev. D 53 (1996) 816.

[11] K. S. Thorne, R. H. Price, and D. A. Macdonald, Black Holes: The Membrane Paradigm (Yale University Press, New Heaven, CT, 1986).

[12] A. Curir, Gen. Rel. Grav. 13 (1981) 417; 13 (1981) 1177.

[13] D. Pavón and J.M. Rubí, Phys. Rev. D 37 (1988) 2052; D. Pavón, Phys. Rev. D 43 (1991) 2495.

[14] R. G. Cai, R. K. Su, and P. K. N. Yu, Phys. Rev. D 48 (1993) 3473; D 52 (1995) 6186; R. G. Cai and R. K. Su, Chinese Phys. Lett. 11 (1994) 526; R. K. Su, R. G. Cai, and P. K. N. Yu, Phys. Rev. D 50 (1994) 2932.

[15] C. F. E. Holzhey and F. Wilczek, Nucl. Phys. B 390 (1992) 447; J. Preskill, P. Schwarz, A. Shapere, S. Trivedi, and F. Wilczek, Mod. Phys. Lett. A 6 (1991) 2353. 
[16] O. Kaburaki, I. Okamoto, and J. Katz, Phys. Rev. D 47 (1993) 2234; J. Katz, I. Okamoto, and O. Kaburaki, Class. Quantum Grav. 10 (1993) 1323.

[17] O. Kaburaki, Phys. Lett. A 185 (1994) 21; Gen. Rel. Grav. 28 (1996) 843.

[18] O. Kaburaki, Phys. Lett. A 217 (1996) 315.

[19] R. G. Cai, Z. J. Lu, and Y. Z. Zhang, Phys. Rev. D 55, (1997) 853.

[20] M. Bañados, C. Teitelboim, and J. Zanelli, Phys. Rev. Lett. 69 (1992) 1849; M. Bañados, M. Henneaux, C. Teitelboim, and J. Zanelli, Phys. Rev. D 48 (1993) 1506.

[21] G. T. Horowitz and A. Strominger, Nucl. Phys. B 360 (1991) 197.

[22] M. J. Duff, R. R. Khuri, and J. X. Lu, Phys. Rep. 259 (1995) 213.

[23] A. Strominger and C. Vafa, Phys. Lett. B 379 (1996) 99; C. G. Callan Jr. and J. Maldacena, Nucl. Phys. B 472 (1996) 591; G. Horowitz, D. Lowe, and J. Maldacena, Phys. Rev. Lett. 77 (1996) 430; G. T. Horowitz and A. Strominger, Phys. Rev. Lett. 77 (1996) 2368; S. S. Gubser, I. R. Klebanov, and A. W. Peet, Phys. Rev. D 54 (1996) 3915; J. M. Maldacena, Nucl. Phys. B 477 (1996) 168: C. G. Callan Jr., J. M. Maldacena, and A. W. Peet, Nucl. Phys. B 475 (1996) 645; I. R. Klebanov and A. A. Tseytlin, Nucl. Phys. B 475 (1996) 164; J. C. Breckenridge, R. C. Myers, A. W. Peet, and C. Vafa, Phys. Lett. B 391 (1997) 93; and references therein.

[24] J. X. Lu, Phys. Lett. B 313 (1993) 29.

[25] G. W. Gibbons, Nucl. Phys. B 207 (1982) 337; G. W. Gibbons and K. Maeda, Nucl. Phys. B 298 (1988) 741.

[26] T. Muto, E-print/hep-th/9605017 (unpublished).

[27] R. G. Cai, R. K. Su, and P. K. N. Yu, Phys. Lett. A 195 (1994) 307.

[28] G. W. Gibbons and S. W. Hawking, Phys. Rev. D 15, (1977) 2752. 
[29] L. Landau and E. M. Lifshitz, Statistical Physics (Pergamon Press, New York, 1958).

[30] I. Okamoto, J. Katz, and R. Parentani, Class. Quantum Grav. 12 (1995) 443; R. Parentani, E-print/gr-qc/9410017 (unpublished).

[31] J. Katz, Mon. Not. R. Astron. Soc. 183 (1978) 765; 189 (1979) 817.

[32] S. W. Hawking, G. T. Horowitz, and S. F. Ross, Phys. Rev. D 51 (1995) 4302.

[33] C. Teitelboim, Phys. Rev. D 51 (1995) 4315.

[34] P. C. Aichelburg and R. Güven, Phys. Rev. D 24 (1981) 2006; D 27 (1983) 456; Phys. Rev. Lett. 51 (1983) 1613; G. W. Gibbons and C. M. Hull, Phys. Lett. B 109 (1982) 190; K. P. Tod, Phys. Lett. B 121 (1983) 241.

[35] O. Coussaert and M. Henneaux, Phys. Rev. Lett. 72 (1994) 183.

[36] G. W. Gibbons, Nucl. Phys. B 207 (1982) 337; G.W. Gibbons and K. Maeda, Nucl. Phys. B 298 (1988) 741; D. Garfinkle, G. T. Horowitz, and A. Strominger, Phys. Rev. D 43 (1991) 3140; A. Shapere, S. Trivedi, and F. Wilczek, Mod. Phys. Lett. A 6 (1991) 2677; L. J. Romans, Nucl. Phys. B 383 (1992) 395; R. Kallosh, A. Linde, T. Ortín, A. Peet, and A. von Proyen, Phys. Rev. D 46 (1992) 5278; T. Ortín, Phys. Rev. D 47 (1993) 3136.

[37] G. W. Gibbons, G. T. Horowitz, and P. K. Townsend, Class. Quantum Grav. 12 (1995) 297.

[38] H. E. Stanley, Introduction to Phase Transitions and Critical Phenomena (Oxford Univ. Press, Oxford, 1971).

[39] D. I. Uzunov, Introduction to the Theory of Critical Phenomena: Mean Field, Fluctuations and Renormalization (World Scientific, Singapore, 1993).

[40] J. Traschen, Phys. Rev. D 50 (1994) 7144. 
[41] I. R. Klebanov and A. A. Tseytlin, Nucl. Phys. B 479 (1996) 319.

[42] D. Garfinkle, G. T. Horowitz, and A. Strominger, Phys. Rev. D 43 (1991) 3140.

[43] M. Cadoni and S. Mignemi, Phys. Rev. D 48 (1993) 5536.

[44] G. T. Horowitz and D. L. Welch, Phys. Rev. Lett. 71 (1993) 328; N. Kaloper, Phys. Rev. D 48 (1993) 2598.

[45] R. G. Cai, Effective spatial dimension of non-dilatonic black p-branes and the description of the entropy on the world volume, E-print/hep-th/9702142, to appear in Phys. Rev. Lett. 